\title{
Rubrics and Their Use in Inclusive Science
}

\author{
KFVIN D. FINSON \\ and Christine K. Ormsief.
}

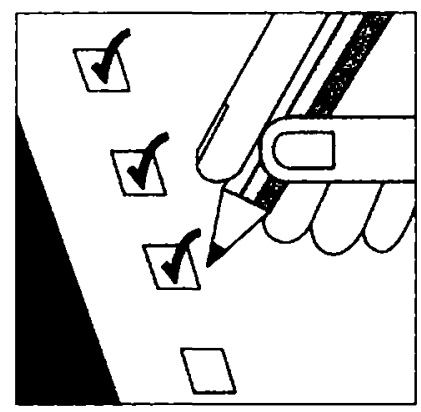

General education teachers are increasingly finding themselves faced with the challenge of providing instruction for all students, including those with learning and behavior problems. Because of the emphasis on experiments and active learning, science classes seem to be one of the most accessible-and accessed-subjects for inclusive programming. However, science instructors are not always as skilled at assessing learning as they are at designing and delivering instruction. A method that can be used to objectively and individually assess the achievement of students with learning and behavior problems is rubrics. When implemented effectively, rubrics can be used to more accurately reflect student achievement and help the educator in determining if remediation is needed.

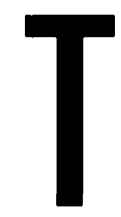

he National Science Teachers Association noted that the U.S. Department of Education (1995) had reported that $35 \%$ of the 4.3 million students with disabilities in this nation's schools were mainstreamed in classrooms. Another $35 \%$ of students with disabilities received some out-of-class instruction from special education teachers. 'The majority of students with learning disabilities (LD) and/or behavior disorders (BD) presently receive much of their academic instruction in general education classrooms (Scruggs \& Mastropieri, 1994; West \& Brown, 1987). In some cases, the inclusion of these students has occasioned some problems, such as preparing general education teachers to use activities, laboratories, and assessments designed for students with exceptional education needs. Students who are not appropriately, actively

engaged in all likelihood will fare poorly in academics (Finson, Ormsbee, Jensen, \& Powers, 1997). Not only must teachers provide the type of learning that will engage their students, they must also develop and use activities and assessments that can be scored so that the results can be used to determine whether continuation or remediation is the next step.

Although the literature contains relatively few studies about the modification of science activities specifically for use with students with LD or BD (Atwood \& Oldham, 1985; Hudson, Graham, \& Warner, 1979; Ormsbec \& Finson, in press; Sullivan \& Petersen, 1986; Wielert \& Sheldon, 1984; Williams \& Algozzine, 1979), there are even fewer concerning scoring criteria, especially as they apply to science taught in the inclusive classroom (Finson, et al., 1997). This despite the fact that considerable literature exists about scoring student work in general education settings.

Scoring criteria are sometimes referred to as "rubrics" and are as important as the assessments or activities themselves. The most effective assessments actually help students learn. The best assessments should flow directly from instruction; the two thus become closely interconnected with regard to learning. In this sense, both instruction (activities) and assessment must engage students.

\section{About Rubrics}

Today, we often hear the term rubric when we are working with assessments, although rubrics can be used for activities as well. A rubric is a guide to follow when scoring assessments or activities. The original definition 
of rubric comes from "inarks in red," a term probably derived from the past as a result of grading student papers. Today, it refers to specific guidelines telling one how to score all of or parts of an assessment or activity. $\Lambda$ variety of such guidelines exist, but they generally come in one of two forms: analytic or bolistic (each will be described in more detail later). Criteria that determine the specific type of rubric designed or selected may include the focus or intent of the assessment, the type of instruction preceding assessment, and personal preferences of the individual doing the assessing.

Rubrics provide detailed breakdowns of points to be awarded for learner responses and, perhaps more importantly, bozi those points should be awarded. Whenever a teacher prepares an assessment, he or she probably has some idea of what is a correct or acceptable answer. In the case of forced-choice assessments, a scoring key is probably made to accompany the assessment. For essay questions, the educator may record the salient ideas or facts a learner must communicate in order to receive a certain number of points. Both examples are essentially rubrics, yet rubrics are more formalized and often provide some preliminary information as to the context in which and conditions under which the assessment is to be administered.

The criteria used in a rubric should be established prior to giving the task to the learner. This is done to avoid problems related to scorer inconsistency; that is, awarding of one set of points for a response and then awarding a different set of points for the same level of response on a different item or from another learner. Establishing the criteria early also helps the person designing the assessment to be certain that he or she is focusing on what he or she intends to focus. If the assessment task and the rubric do not match, then an obvious problem will be encountered when scoring the product. Designing an appropriate rubric is just as important as designing the assessment task, and it sometimes requires comparable time and effort. As with assessments, rubrics may require revision after they are first used in order to best measure student performance. An example of such an instance would be when the rubric's design fails to account for the range of possible responses that learners provide. 'This is particularly important when assessing products of individuals with disabilities.

Once the scoring criteria are established, the assessment or activity can be administered. For the most effective use, the person assessing the students should make sure that each learner is aware of and understands exactly what is required to achieve a specific score on each task. After the assessment is administered, each learner response is compared to an example of an acceptable response on the rubric. A total, or overall score, is then derived for the assessment. When used consistently in this way, rubrics can serve the dual purpose of assess- ment tool and vehicle for effective teaching. As assessment tools, rubrics are standards against which a learner's work can be compared and judged. As a teaching tool, rubrics can be goals for learner progress and performance. When students are well aware of the levels of task completion expected prior to the assignment, they are much more likely to complete the tasks successfully. In addition, rubrics can be used to assess the efficacy of a particular curriculum; hence, the intent of an assessment and its rubric must be clearly established at the outset.

\section{Types}

ANALYTIC Rubrics. Analytic rubrics are used to award points for very specific responses on different portions of an assessment. All points from each part of the assessment are added together to derive an overall score and determine the level of student performance. Scoring criteria are quite specific as to how points can and cannot be awarded. Persons using analytic rubrics must critique each response made by a student and score the response according to the established criteria; thus, there is very little room for subjectivity. Because analytic rubrics are more process oriented than product oriented, they may be preferable for use with students with LD or BD. Analytic rubrics may be restrictive or may allow for variability in student responses. For example, a student may complete a task demonstrating her ability to accurately determine the mass of an object where the correct answer is $\mathbf{4 5}$ grams. An example of a restrictive analytic rubric applied to such a task would be:

- 1 point = Student determines mass of object at exactly 45 grams.

- 0 points = Student determines mass of object to be other than 45 grams.

\section{OR}

- Student does not attempt to fill the cylinder and/or fails to record an answer.

In a more flexible application of the analytic rubric, the primary instructional objective may be modified due to the quality of the balance the student uses or consideration of other noninstructional issues such as time availability or student ability. In this case, the teacher may wish to use a rubric that uses error ranges designated by percentages or actual measurement values. Each such measuring activity included within the assessment would have specific scoring criteria. If there are three things the student must measure, then each will have a range of possible answers and accompanying points. For example:

- 3 points $=$ Student states the object's mass to be $4+46$ grams. 
- 2 points $=$ Student states the object's mass to be $42-43$ grams or $47-48$ grams.

- 1 point = Student states the object's mass to be less than 42 grams or greater than 48 grams.

- 0 points $=$ Student does not attempt to determine the object's mass and/or fails to record an answer.

Holistic Rubrics. Holistic rubrics are used when the teacher wants to assess the overall quality of a student's response. Iolistic rubrics are more product oriented than process oriented, and are primarily concerned with the total performance or product rather than with the individual steps taken to arrive at the final product, as is done with analytic scoring. Criteria for holistic scoring should reflect specific and important elements of a solution to a problem (or to other acceptable answers). When developing holistic rubrics, teachers often find it useful to first determine performance indicators (e.g., superior, acceptable, inadequate, or unacceptable levels). In its purest form, a holistic rubric is not used to award points; instead, student products (or assessments) are simply rated according to the designated indicators. However, because most assessments used in schools lead to evaluation, points can be designated for each indicator. For example:

- 3 points for superior product or solution

- 2 points for acceptable product or solution

- 1 point for inadequate product or solution

- 0 points for no attempt (unacceptable)

It must be emphasized here that relying only on the four points in the above example is insufficient for true holistic scoring. The examiner must include more detail describing each level of performance. For example, the rubric in the sidebar could be used with a student assigned to design and conduct an experiment.

Some educators/assessors find it helpful to prepare a grid or matrix to represent a holistic rubric. The indicators are usually arranged across the top, and specific performances are arranged in a column along the left. Descriptions are provided in each box of the grid. Such grids have the advantage of allowing the teacher to create a rather comprehensive holistic rubric that encompasses several performances and at the same time alleviates the need for multiple pages for the rubric.

\section{Use in Inclusive Classrooms}

An analytic rubric may be most appropriate to use with learners who have exceptional education needs. 'These learners can receive credit for the process skills required to complete the task rather than simply being restricted to the product itself. Analytic and holistic rubrics can be used together, as they measure different aspects of a task (one the process and the other the product). A science experiment might be scored holistically for all learners as

\section{Example of a Holistic Rubric}

3 Points-Superior Performance

- Hypothesis is clearly stated and is limited to the variables involved.

- Variables are correctly identified and none are misidentified.

- Procedures are clearly stated-including safety considerations, materials list is complete, and controls are clearly and correctly identified.

- Conclusions-including analysis and interpretation of data - are thorough and take into account all data, are based on sound scientific principles that apply to the experiment, and are not overgeneralized or unclear.

\section{Points-Acceptable Performance}

- The hypothesis is somewhat clear but may include some extraneous variables or may omit needed phrasing about one variable.

- All but one variable are correctly identified.

- Most necessary procedures are clearly described but may include one or two minor extraneous steps, have unclear safety considerations, have incomplete materials lists, and/or have a control that includes a minor omission or addition.

- The conclusion takes into account most data and tends to be based on scientific principles but may overgeneralize to a slight extent.

\section{Point-Poor Performance}

- Hypothesis is somewhat unclear and fails to include most variables involved; it may even include some extraneous variables.

- Two or more variables are not correctly identified, and/or other variables are misidentified.

- Few procedures are stated or procedures are stated unclearly, the steps include too many unnecessary steps or fail to include those needed to complete the experiment, the materials list is incomplete or includes many unnecessary items, and the control is not clearly described and may or may not include omissions or additions.

- The conclusion fails to take into account major portions of data or attempts to include data not obtained directly from the experiment, and/or tends to overgeneralize, or misapplies scientific principles.

(sidebar continues) 
(contimued from p. 81)

\section{Points-Not Applicable or No Attempt Is Made}

- Hypothesis has little or nothing to do with the experiment, or no attempt is made to make a hypothesis.

- Variables identified have little or nothing to do with the experiment or are misidentified; no attempt is made to identify and deal with variables.

- Procedures given are vague/unclear or do not address experiment; materials listed are insppropriate, too few, or include many unnecessary items; control is absent or inappropriate for experiment; no attempt is made to describe procedures.

- Conclusion does not address the data collected, ignores data and/or focuses on data external to the experiment, misapplies scientific principles; no attempt is made to analyze or interpret data and draw conclusions.

a screening procedure to determine overall quality of the product. As a follow-up, the teacher might use an analytic rubric to assess the work of the learners who did not produce an experiment with satisfactory results.

Establishing rubric criteria can be difficult. Some teachers feel compelled to fit any and all scoring into a certain scheme, such as having all questions worth 10 points. If only 5 points are justified by the rubric, then imposing a 10-point scale is inappropriate. Typically, and particularly for analytic rubrics, three to six categories or levels of response are common. Learners in lower grades may require rubrics with fewer response levels.

Teachers of students with exccptional learning needs may find a 3-point rubric to be most useful. 'This rubric is rather casy to write because it is based on the scale of above average, average, and below average responses. In a 3 -point rubric, the top score is given to students who demonstrate an exceptional understanding of the component of the task being analyzed, the middle score is allotted to students who show a satisfactory understanding, and the lowest score is given to students who demonstrate an incomplete understanding or misconceptions about the task component. The distinction between the three response levels is easily defined and recognized.

Some teachers may use a 4-point rubric because the 4 points translate easily into the traditional A, B, C, and D grade categories. Every task that is scored does not necessarily have to be uniform in terms of each item having the same number of response levels as all other items. In other words, one task may have three response levels whereas another will have five. Teachers can choose the number of points on the rubric based on the needs of the particular learners and the task being assessed.

\section{Practice in Applying a Rubric}

Sometimes the difference between assessment and cvaluation is not clear. Even though the distinctions may seem obvious on the surface, educators may experience difficulties in the application. In this section, an example of an assessment activity-_Rubber Band Stretch"-is provided to show (a) how to apply a rubric and (b) how assessment is different from evaluation.

In practice, the Rubber Band Stretch assessment would be administered to students after instruction was provided on measuring, hypothesizing, graphing, predicting, identifying and controlling variables, and on evaluating the elasticity of various materials. Figure 1 provides an analytic rubric for scoring the assessment activity. Figure 2 shows some background information the teacher gathered regarding the assessment and scores obtained by all students in the class. The Rubber Band Stretch forms are completed by two students, Michelle and Heath. Each step is described in detail to show how rubrics are used. 'The analytic rubric provided can be used to score both Michelle's and I Ieath's forms (see Figures 3 and 4).

By examining the two student's completed forms and applying the criteria in the rubric, the teacher can assess the student's work. From this assessment, a general idea of the level of understanding each student possesses about the process skills and concepts taught can be objectively identified. Specific outcomes that can be measured include

1. If the student knew how to correctly construct a data table,

2. How to graph data from a data table,

3. How to interpret that graph,

4. If the student understands how to formulate a good hypothesis,

5. How to identify specific types of variables, and

6. If the student can expand upon what he or she learned from the activity and project that thinking to future possibilities.

Other possible questions a teacher may want to consider:

1. Overall, how would one summarize Michelle's grasp of the process skills and concepts?

2. How would one summarize Heath's?

3. Overall, were either or both students successful:

4. What do the results suggest in terms of instruction: Is it time to move on to the next unit, or does the student require remediation?

5. Would such results require reconsideration of how instruction was delivered prior to the assessment? 


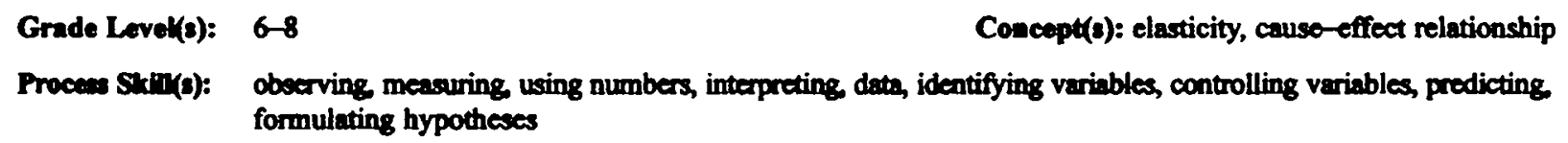

Objective(s): Students will be able to make an accurate prediction based upon data collected and patterns observed in the stretching of a rubber band, and they will be able to generate a hypothesis useful for further testing.

\section{Queation *}

$$
\text { Criteria }
$$

46: Dare Thile

Sadent corroctly constructs data table with proper column beadings and units of measurement, has all data for one variable 4 reconded in either ascending or descending onder and all datn for other variable arminged so as to correlate with the first variable's daten.

Student correctly constructs data table with proper column headings and units of measurement, but recording of data is 3 not in ascending or descending onder for either variable.

Student correctly constructs dable with proper column headings and units of measurement, but has serious errors in 2 reconding of datu OR has correct deta but fails to include proper column headings and/or units of measurement.

Student has significant errors in construction of data table and in reconding of data.

Student does not attempt.

7: Griph

Student constructs graph correctly with all axes correctly divided, increenents on axes proportional, increments reflecting 4 total range of date, axes labeled correctly with values and measuring units, all dath points correctly plotted and connected with a line.

Student constructs graph as above but with one or two minor errors, such as axis increments not being proportional throughout axis, omission of measuring units, incorrect plotting of date, omission of groph line connecting points, etc.

Student constructs graph but has three or more minor errors as described above.

Student constructs graph that contains significant errors.

Student constructs inappropriate gruph, graphs irrelevant data, or does not attempt.

8 Student generates hypothesis that is general in its wonding and takes into account the interrelationship of the variables involved. (Example!. The amount of stretch of a rubber band will increase with unequal but langer lengths as equal amounts of weight are added to it").

Student gexerates hypothesis that is not general in its wording, but takes into scoount the interrelationship of the variables involved. (Example: "This rubber band stretched in increasing amounts as more weights were hung on it") OR generates hypothesis thet is general in wording but fails to take into account the interrelationship of the variables involved (Example: "The amount of stretching of a rubber band gets longer when weight is on it").

Student generutes hypothesis that is not general in its wonding and fails to account for the interrelationship of the variables 1 involved. (Example: "My rubber band got longer").

Student generates a hypothesis that is not related to the activity OR does not attempt.

9 Student correctly determines rubber band length with $0 \%$ error.

Student determines rubber band length with 1-5\%.

Student determines rubber band length with 6-10\% error.

Student error is greater than $10 \%$ or student does not attempt.

10 Student correctly determines number of washers with $0 \%$ error.

Student determines number of washers with 1-5\% error.

Student determines number of washers with 6-10\% error.

Student error is greater than $10 \%$, or student does not attempt. 


\section{Queation *}

11 Student correctly identifies weight (or number of washers) as the independent variable and rubber band stretch as the dependent variable.

Student correctly identifies one of the two variables but fails to correctly identify the second.

Student fiils to correctly identify either variable OR does not attempt.

12 Student correctly names two variables. For example: rubber band age, rubber band thickness, type of rubber in rubber band, number of times rubber bend has been used, rubber band width, etc.

Student correctly names only one variable.

- For this activity, the students were to use the same rubber band.

- The teacher pretested the rubber band's elasticity as described in the instructions. The teacher's pretest results are as follows:

\begin{tabular}{|c|c|}
\hline \# Washers & $\begin{array}{c}\text { Rubber Band } \\
\text { Length (cm) }\end{array}$ \\
\hline 0 & 8 \\
2 & 10 \\
4 & 15 \\
6 & 22 \\
8 & 27 \\
\hline
\end{tabular}

- The teacher graphed the data and used the graph for Questions 8 and 9. The graph is reproduced below:

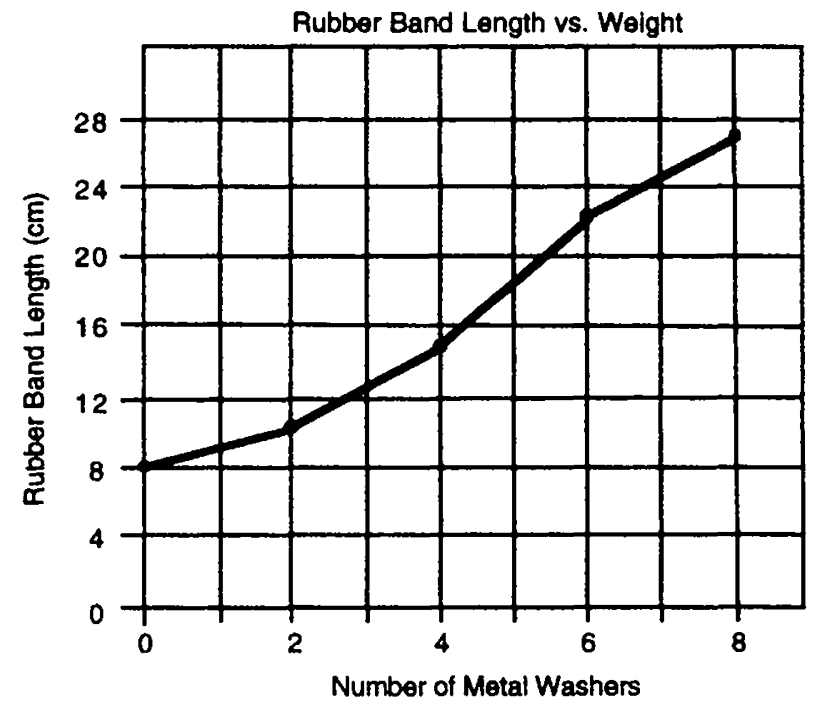

\begin{tabular}{|c|c|c|c|c|c|}
\hline CLASS & SCORES & ON RUBBER BAND & STRETCH (21 & points p & osslble) \\
\hline TUDENT & SCORE & $\%$ OF TOTAL & STUDENT & ScQE & \% OF TOT \\
\hline Shannon & 20 & 95 & Nancy & 14 & 67 \\
\hline Mark & 20 & 95 & Ben & 13 & 62 \\
\hline Deanna & 19 & 90 & Thomas & 12 & 57 \\
\hline Jill & 17 & 81 & Marcio & 12 & 57 \\
\hline John & 16 & 76 & Donnie & 11 & 52 \\
\hline Matt & 16 & 76 & James & 9 & 43 \\
\hline Jason & 15 & 71 & Elizabeth & 9 & 43 \\
\hline Meg & 15 & 71 & Danielle & 8 & 38 \\
\hline Carrio & 15 & 71 & Steven & 6 & 29 \\
\hline Chris & 14 & 67 & Randy & 5 & 24 \\
\hline
\end{tabular}

Figure 2. Background Information for Rubber Band Stretch. 




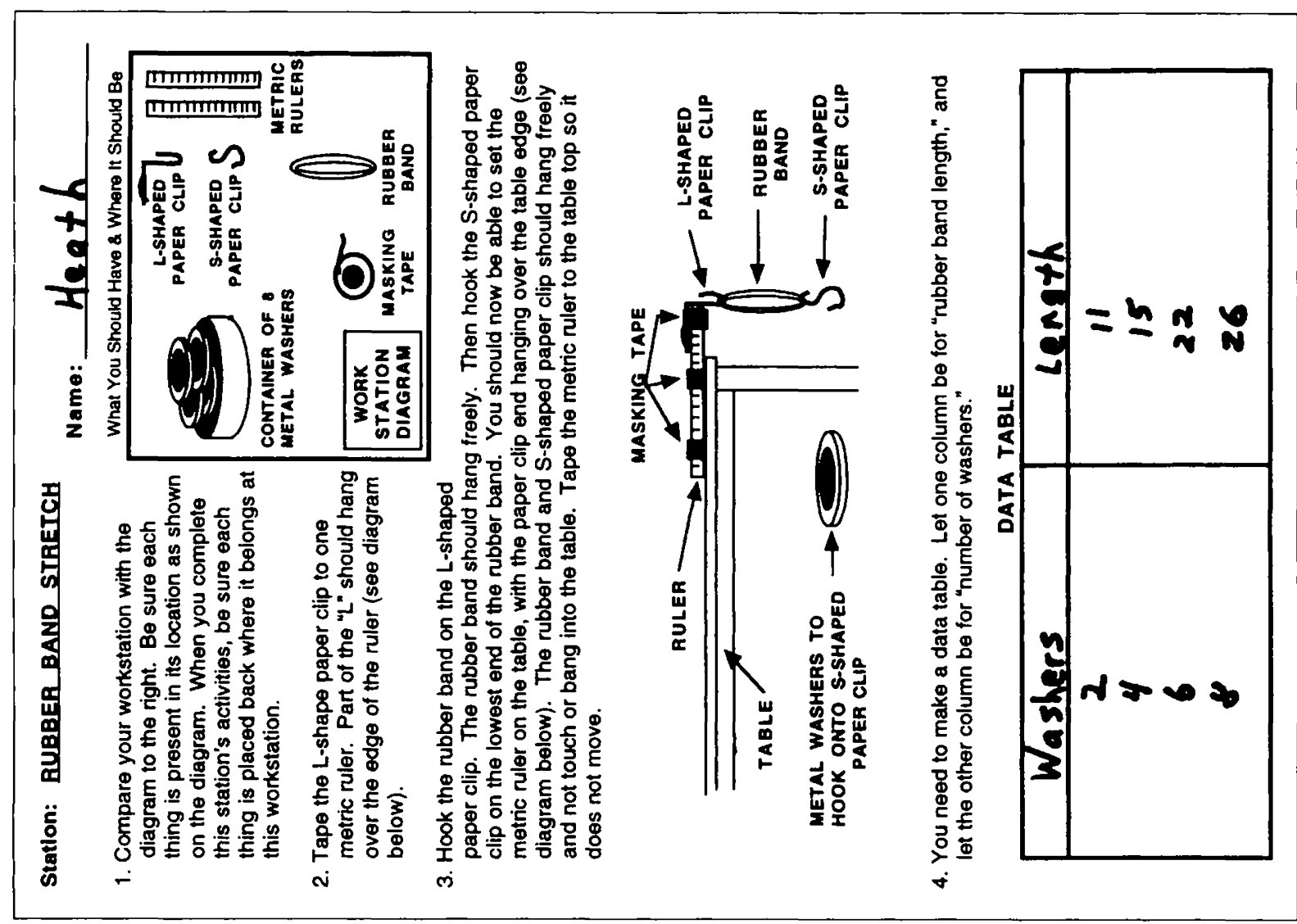

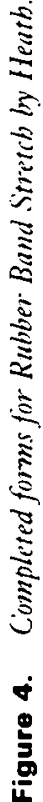

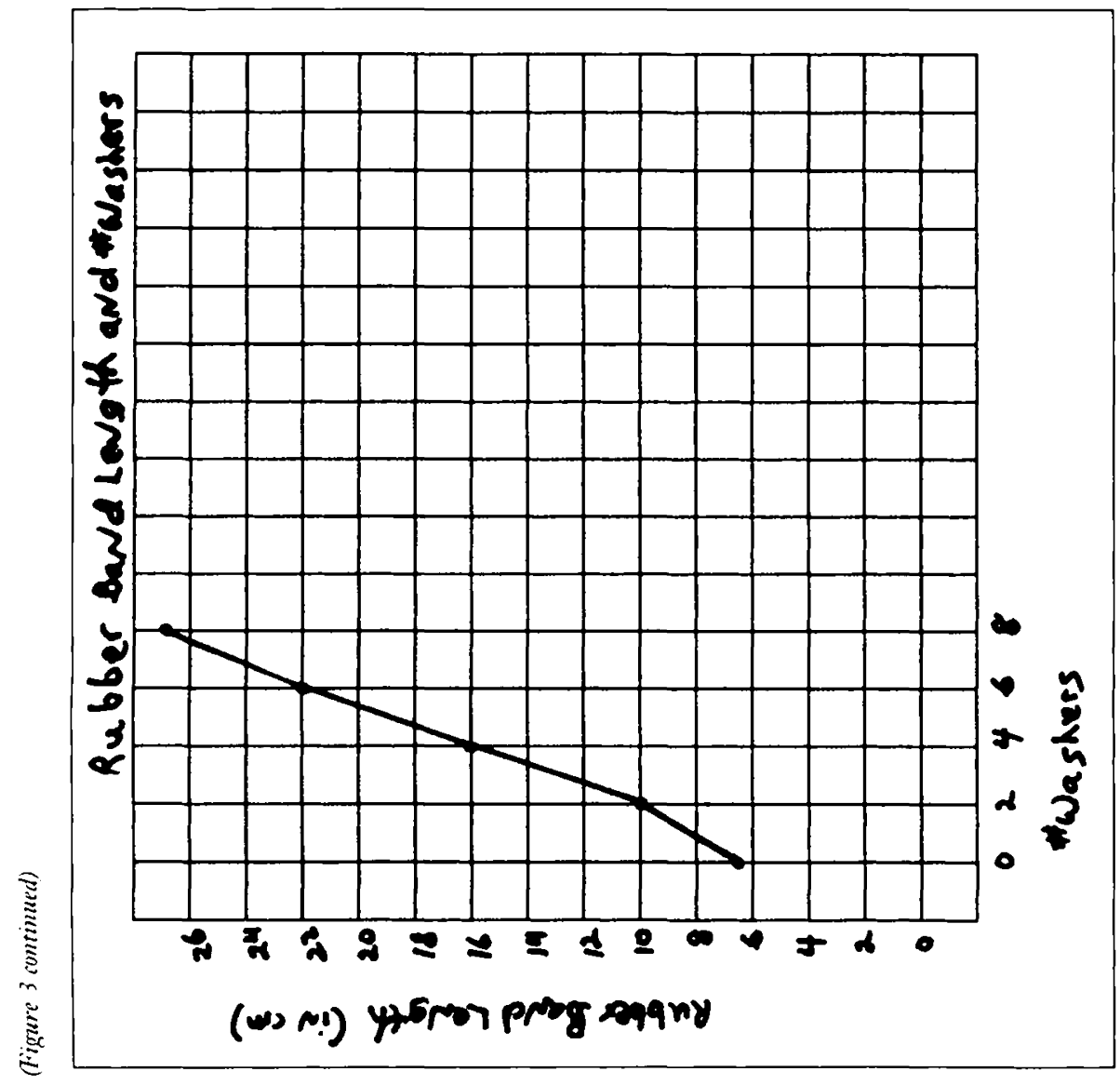



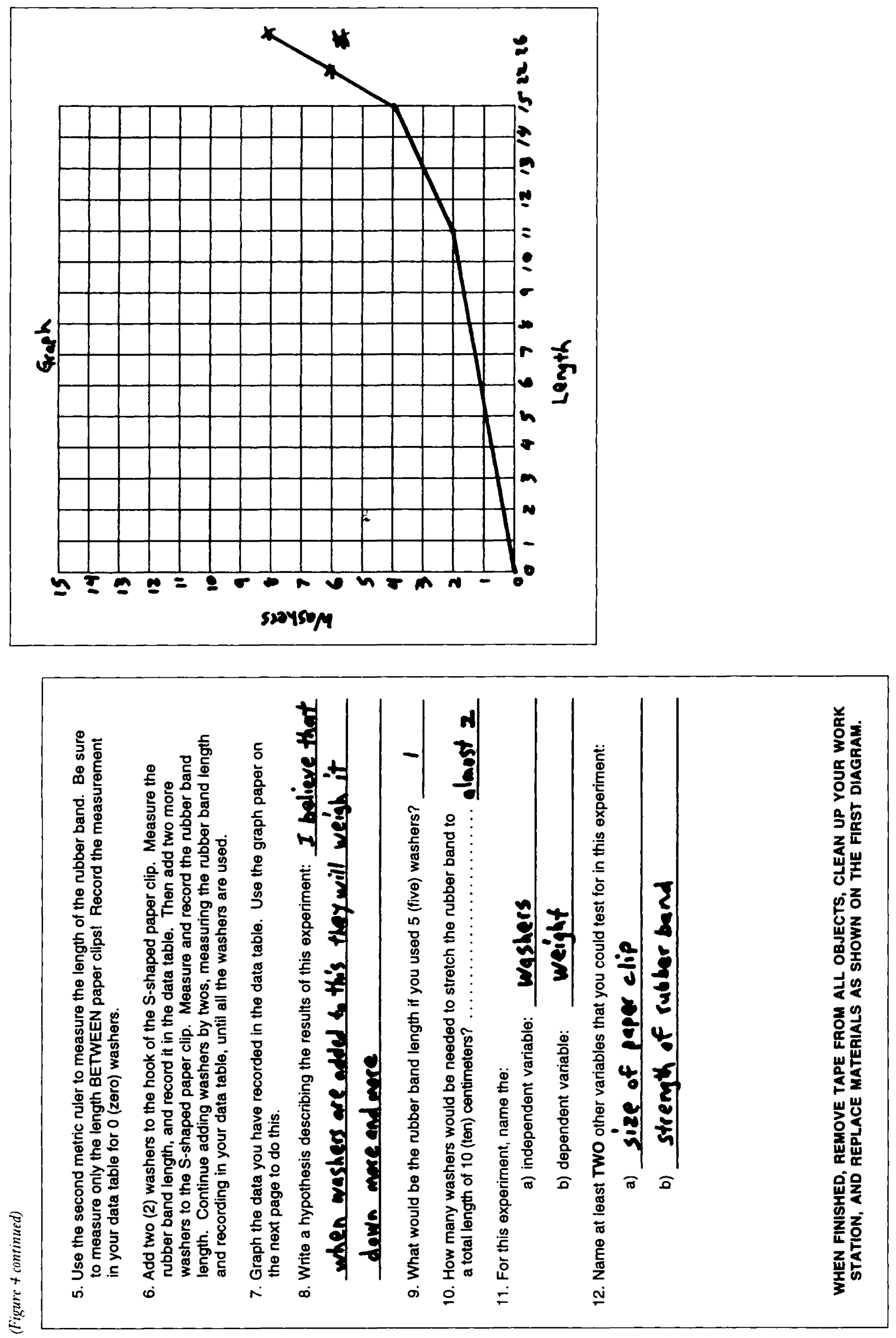
After the rubric has been applied to assess the work of Michelle and Heath, the results need to be evaluated. Figure 3 shows how other students in the class performed on the assessment. The teacher should now determine the following two things:

1. Where do Michelle and I leath fall within the student rankings?

2. What letter grades will be assigned to each student?

The grading scale may be one specified by a school or district, or some other grading-scale criteria may be used.

The process of ranking Michelle and Heath among other students, and of assigning grades, is called evaluation. In this process, students' assessment scores are compared with some standard (e.g., a grading curve derived from all students' scores, a grading scale imposed by the school district). With standardized tests, the students' scores would be compared to the scores of literally thousands of other students. Whatever the standard selected for comparison, Michelle's and Heath's work would be placed somewhere on the continuum between those of the highest-performing student and the lowestperforming student. Based on the evaluation, new instructional decisions should be made. Consider the following questions:

1. Do Michelle and Heath qualify to proceed on to the next unit:

2. What grades will be recorded on their report cards?

3. Or, more importantly, do they qualify to graduate to the next higher grade level?

4. Do either require remediation, and if so, what should it entail?

Assume for a moment that Michelle is an exceptionally bright student who seems to learn material effortlessly. She does quite well on objective tests in particular. How might Michelle's evaluation change, if it were to change at all? Assume further that Heath has a severe learning disability and that when he entered the classroom at the beginning of the year, he experienced great difficulty handling laboratory equipment and had problems completing measurements with any accuracy. How might this information affect an instructor's perceptions of Heath's achievement on this task and overall progress in the course? Perhaps the teacher might determine that Michelle really did not demonstrate any significant learning, whereas Heath showed tremendous growth.

\section{Conclusion}

The implications for developing curricula, assessments, and rubrics specifically targeting inclusive class- room instruction are clear. The design and use of quality rubrics is essential in determining the success of student learning and teacher instruction. Using carefully designed rubrics will help teachers make appropriate curriculum and assessment changes and increase the chances of success for students from special populations, particularly in areas such as science.

\section{About the Authors}

Kevin D. Finson, $\mathrm{PhD}$, is a professor of science education at Western Illinois University in Mlacomb. His current research interests include children's perceptions of scientists and modifing science activities and assessments for use in mainstreamed classrooms. Christine $\mathbf{K}$. Ormsbee is an associate professor at The L'niversity of Oklahoma in the Department of Fiducational Psychology. She also serves as ficld experience coordinator for undergraduate and graduate preservice special educators. I ler research interest focuses on preassessment and general education interventions for children at risk for school failure. Address: Kevin D. Finson, Department of Flementary Fducation and Reading, Western Illinois University, 1 University (Gircle, Macomb, IL 61455 .

\section{References}

Atwood, R. K., \& Oldham, B. R. (1985). 'Teachers' perceptions of mainstreaming in an inquiry oriented elementary science program. Science Education, 69, 619-624.

Finson, K. D., Ormsbee, C. K., Jensen, M., \& Powers, D. T. (1997). Science in the mainstream: Retooling science activities. Foumal of Science 'Teacher Fiducation, 8(3), 219-232.

Hudson, F., Graharn, S., \& Warner, W. (1979). Mainstreaming: In examination of the attitudes and needs of regular classroom teachers. I.earning Disability Quarterly, 2, 58-62.

National Science 'Teachers Association. (1994, May/June). IST:1 Repont [Newspaper]. Washington, DC: Author. (Available from NSTA, 1840 Wilson Blvd., Arlington, VA 22201-300())

Ormsbec, C. K., \& Finson, K. D. (in press). Modifying science activities and materials to enhance learning for students with learning and behavior problems. Interzention in School and Clinic.

Scruggs, T. F., \& Mastropieri, M. A. (199)). Refocusing microscope activities for special students. Science Scope. 17(6), $74-78$.

Sullivan, M. J., \& Petersen, B. (1986). Recognizing the superior sitence teacher of the bandicapped. Carbondale, IL: Southern Illinois University. (ERIC Document Reproduction Service No. EI) 271 301)

U.S. Department of Fducation. (1995). Anmual report to Congress on the implementation of IDEA 1 . Washington, DC: Author.

West, J. F., \& Brown, P. (1987). State departments of education policies on consultation in special education: The state of the states. Remedial and Special Fducation, 8(3), 45-51.

Wielert, J. S., \& Sheldon, D. S. (1984). Make science accessible to all your students. The Silence Teacher; 5l(4), 56-58.

Williams, R. J., \& Algozzine, B. (1979). Teachers' attitudes touard mainstreaming. The Elementary School Journal, 80(2), 63-67. 